JIRSS (2021)

Vol. 20, No. 01, pp 219-246

DOI:10.52547/jirss.20.1.219

\title{
Stress-Strength and Ageing Intensity Analysis via a New Bivariate Negative Gompertz-Makeham Model
}

\author{
Hossein-Ali Mohtashami Borzadaran ${ }^{1,2}$, Hadi Jabbari ${ }^{1}$, Mohammad Amini ${ }^{1}$, and \\ Ali Dolati ${ }^{1}$ \\ ${ }^{1}$ Department of Statistics, Ordered Data, Reliability and Dependency Center of Excellence, \\ Ferdowsi University of Mashhad, Mashhad, Iran. \\ ${ }^{2}$ Sun-Air Research Institute, Ferdowsi University of Mashhad, Mashhad, Iran.
}

Received: 28/11/2020, Revision received: 07/02/2021, Published online: 03/04/2021

\begin{abstract}
In Demography and modelling mortality (or failure) data the univariate Makeham-Gompertz is well-known for its extension of exponential distribution. Here, a bivariate class of Gompertz-Makeham distribution is constructed based on random number of extremal events. Some reliability properties such as ageing intensity, stress-strength based on competing risks are given. Also dependence properties such as dependence structure, association measures and tail dependence measures are obtained. A simulation study and a performance analysis is given based on estimators such as MLE, Tau-inversion and Rho-inversion.
\end{abstract}

Keywords. Bivariate Exponential Distribution, Demography, Survival Function, Hazard Function.

MSC: 39A60, 62E10, 62H10.

Hossein-Ali Mohtashami Borzadaran (h.mohtashami@mail.um.ac.ir)

Hadi Jabbari (jabbarinh@um.ac.ir)

Corresponding Author: Mohammad Amini (m-amini@um.ac.ir)

Ali Dolati (dolati50@yahoo.com) 


\section{Introduction}

The estimation and calculation of mortality tables are of great importance in Actuary. One of the first leading models in this area was the famous Gompertz distribution (Gompertz (1825)) which is a truncated extreme value distribution and flexible in the right-hand tail of distribution (Johnson et al. 1994, pp. 25, 640 and Wang et al. 1998). This univariate distribution is considered as an generalization of exponential distribution since it can be obtained from limits of sequences from Gompertz distributions.

The univariate Gompertz-Makeham distribution has gained the attention of many researchers. Juckett and Rosenberg (1993) explained the advantages of the Gompertz distribution to Weibull distrbution. An MCMC algorithm to generate data from Gompertz-Makeham distribution was obtained by Scollnik (1995). An efficiency comparison of Gompertz, Gompertz-Makeham and Lee-Carter mortality models for risk management is given by Melnikov and Romaniuk (2006). Feng and He (2008) used the least square method to estimate the parameters of Gompertz-Makeham distribution. Missov and Lenart (2013) derived a closed form solution to the life expectancy integral corresponding to the cases of homogeneous and gamma-heterogeneous populations. Abd El-Bar (2018) presented the transmuted Gompertz-Makeham model.

The negative Gompertz-Makeham distribution was defined by Marshall and Olkin (2007) and named as the negative Gompertz-Makeham distribution with survival function

$$
\bar{F}(t)=\exp \left\{-\tau \theta \lambda t+\theta\left(e^{-\lambda t}-1\right)\right\},
$$

where $\lambda, \tau, \theta, t>0$. Also, this model is constructed from a sequence of random variables. Let $\left\{X_{i}, i \geq 0\right\}$ be independent sequence of exponential distribution such that $X_{0} \sim \operatorname{Exp}(\delta)$ and $X_{i} \sim \operatorname{Exp}(\lambda)$ for $i \geq 1$ and $N$ be a Poisson random variable with parameter $\theta$. Then the survival function of $Y=\min \left(X_{0}, X_{1}, \ldots, X_{N}\right)$ is the negative Gompertz-Makeham distribution denoted by $\operatorname{NGM}(\delta, \lambda, \theta)$ with survival function

$$
\bar{F}(t)=\exp \left\{-\delta t-\theta\left(1-e^{-\lambda t}\right)\right\} ; \quad \lambda, \delta, \theta, t>0 .
$$

By reparameterizing $\delta=\tau \theta \lambda$ where $\tau>0$, we derive the model

$$
\bar{F}(t)=\exp \left\{-\tau \theta \lambda t-\theta\left(1-e^{-\lambda t}\right)\right\} ; \quad \lambda, \tau, \theta, t>0 .
$$

The function in (1.2) is actually the survival function of $Z=\min (X, Y)$ where $X$ and $Y$ are independent with $X$ having a negative Gompertz distribution, and $Y$ having 
an exponential distribution (Marshall and Olkin 2007). The given model has been applied by Bailey and Homer (1977) and Bailey et al. (1977) in the context of kidney transplantation where they used the three parameter hazard rate of (1.2).

Moreover, the multivariate extension of these models have been studied. Brockett (1984) derived a bivariate Gompertz distibution by functional equation approach. Adham and Walker (2001) introduced a multivariate Gompertz distribution using frailty models. El-Sherpieny et al. (2013) proposed a bivariate Gompertz MarshallOlkin model. Marshall and Olkin (2015) derived the Gompertz-Makeham distribution using a limiting case of Kaminsky's functional equation. Kolev (2016) characterized a class of bivariate Gompertz distributions.

In the following, some concepts of dependence and ageing notions obtained from Nelsen (2007) and Lai and Xie (2006) are introduced:

- The joint random variable $(X, Y)$ is said to be positive quadrant dependent (PQD) if for every distribution function $F$, we have $F_{X, Y}(x, y) \leq F_{X}(x) F_{Y}(y) ; \forall x, y$.

- The joint random vector $(X, Y)$ is said to be right corner set increasing (RCSI) if $P\left(X>x, Y>y \mid X>x^{\prime} Y>y^{\prime}\right)$ is increasing in $x^{\prime}$ and $y^{\prime}$ for all $x$ and $y$.

- A bivariate function $f: R^{2} \rightarrow R$ is totally positive of order two (TP2) if for every $x \leq x^{\prime}$ and $y \leq y^{\prime}$ we have $f(x, y) f\left(x^{\prime}, y^{\prime}\right)-f\left(x^{\prime}, y\right) f\left(x, y^{\prime}\right) \geq 0$.

- For every pair $(X, Y)$ with bivariate survival function $\bar{F}$ and marginals $\bar{F}_{1}$ and $\bar{F}_{2}$, two famous measures of association are Kendall's Tau $\tau=4 \int_{(0, \infty)^{2}} \bar{F}(x, y) d \bar{F}(x, y)-1$ and Spearman's Rho $\rho_{s}=12 \int_{(0, \infty)^{2}}\left(\bar{F}(x, y)-\bar{F}_{1}(x) \bar{F}_{2}(y)\right) f_{1}(x) f_{2}(y) d x d y$.

- For every pair $(X, Y)$ with distribution function $F$ and marginals $F_{1}$ and $F_{2}$ the lower and upper tail dependence coefficients $\lambda_{L}$ and $\lambda_{U}$ are defined as $\lambda_{L}=$ $\lim _{t \rightarrow 0^{+}} P\left(X \leq F_{1}^{-1}(t) \mid Y \leq F_{2}^{-1}(t)\right)$ and $\lambda_{U}=\lim _{t \rightarrow 1^{-}} P\left(X>F_{1}^{-1}(t) \mid Y>F_{2}^{-1}(t)\right)$.

- The joint random vector $(X, Y)$ is said to have bivariate lack of memory property whenever it satisfies the relation

$$
P(X>x+t, Y>y+t \mid X>s, Y>t)=P(X>x, Y>y), x, y, t>0 .
$$

- Let the joint random vector $(X, Y) \sim H$. Then

- $(X, Y)$ is said to be bivariate new worse than used of type I (bivariate new better than used of type I) denoted by BNWU-I (BNBU-I), whenever for all $x, y, t>0$

$$
\bar{H}(x+t, y+t) \geq(\leq) \bar{H}(x, y) \bar{H}(t, t) .
$$


- $(X, Y)$ is said to be bivariate new worse than used of type II (bivariate new better than used of type II) denoted by BNWU-II (BNBU-II), whenever for all $x, y, t_{1}, t_{2}>0$

$$
\bar{H}\left(x+t_{1}, y+t_{2}\right) \geq(\leq) \bar{H}(x, y) \bar{H}\left(t_{1}, t_{2}\right) .
$$

In this paper, we are focused on introducing bivariate and multivariate extensions of negative Gompertz-Makeham distribution in (1.1) with decreasing hazard rates. The introduced model is considered for an analysis of ageing concepts and stressstrength index. Also, the need for more mortality models with decreasing failure rates is expressed by Bebbington et al. (2014) since countries like USA, Japan and Sweden have decreasing mortality beyond some point. So, the main purpose of this paper is to introduce a bivariate class of Gompertz-Makeham distribution with decreasing hazard rate using same sequences of random variables.

In Section 2, the bivariate mortality distribution is constructed with a lifetime interpretation. In section 3, dependence properties of the model is presented. Section 4 provides some properties of univariate and bivariate hazard components for the proposed model. Section 5, investigates the notion of Ageing intensity in the univariate and bivariate cases. In section 6, we calculate the stress-strength parameter based on competing risks. In section 7 , we give algorithm to generate data from the presented model and illustrate different plots to see the behaviour of the model. Also, we present three estimators for the dependence parameter and give a performance analysis for the mentioned estimators.

\section{Derivation of the Model}

Suppose we have two sets of $i$ id survival events (for example, failure time) $\left\{X_{i} ; i \geq 0\right\}$ and $\left\{Y_{j} ; j \geq 0\right\}$. Let $\left(N_{1}, N_{2}\right)$ be the counts of these two survival events. So, the joint lifetime of $\left\{X_{i} ; i \geq 0\right\}$ and $\left\{Y_{j} ; j \geq 0\right\}$ is $\left(\min \left\{X_{0}, \ldots, X_{N_{1}}\right\}, \min \left\{Y_{0}, \ldots, Y_{N_{2}}\right\}\right)$. Based on Marshall and Olkin (1997), we present the bivariate extension of negative GompertzMakeham distribution given in (1.1). Consider the sequences of independent random variables $\left\{X_{i} ; i \geq 0\right\}$ and $\left\{Y_{j} ; j \geq 0\right\}$ such that $X_{0} \sim \operatorname{Exp}\left(\alpha_{0}\right), Y_{0} \sim \operatorname{Exp}\left(\beta_{0}\right), X_{i} \sim \operatorname{Exp}\left(\alpha_{1}\right)$ and $Y_{j} \sim \operatorname{Exp}\left(\beta_{1}\right)$ for $i, j \geq 1$. Define

$$
T=\min \left\{X_{0}, \ldots, X_{N_{1}}\right\} \text { and } S=\min \left\{Y_{0}, \ldots, Y_{N_{2}}\right\},
$$

where $\left(N_{1}, N_{2}\right)=\left(P_{1}+P_{12}, P_{2}+P_{12}\right)$ is a bivariate discrete Poisson random vector constructed from three independent Poisson random variables such that $P_{1} \sim P\left(\theta_{1}\right)$, 
$P_{2} \sim P\left(\theta_{2}\right)$ and $P_{12} \sim P(\theta)$. The probability generating function of $\left(N_{1}, N_{2}\right)$ is

$$
A_{N_{1}, N_{2}}(t, s)=\exp \left\{-\theta_{1}(1-t)-\theta_{2}(1-s)-\theta(1-t s)\right\} ; \quad \theta_{1}, \theta_{2}, \theta>0 .
$$

Let $\theta_{1}, \theta_{2} \rightarrow 0$. So, The probability generating function of $\left(N_{1}, N_{2}\right)$ becomes

$$
A_{N_{1}, N_{2}}(t, s)=\exp \{-\theta(1-t s)\} ; \quad \theta>0 .
$$

So, the aim of this section is to find the survival function of $(T, S)$ assuming that $\left\{\left(X_{i}, Y_{j}\right) ; i, j \geq 0\right\}$ is independent of $\left(N_{1}, N_{2}\right)$. Thus,

$$
\begin{aligned}
\bar{F}_{T, S}(t, s) & =P\left(X_{0}>t\right) P\left(Y_{0}>s\right) \sum_{n_{1}=0}^{\infty} \sum_{n_{2}=0}^{\infty}\left\{P\left(X_{1}>t\right)\right\}^{n_{1}}\left\{P\left(Y_{1}>s\right)\right\}^{n_{2}} P\left(N_{1}=n_{1}, N_{2}=n_{2}\right) \\
& =P\left(X_{0}>t\right) P\left(Y_{0}>s\right) A_{N_{1}, N_{2}}\left(P\left(X_{1}>t\right), P\left(Y_{1}>s\right)\right) .
\end{aligned}
$$

Thus, by plugging the probability generating function in (2.2) into (2.3), we get

$$
\bar{F}(t, s)=\exp \left\{-\alpha_{0} t-\beta_{0} s-\theta\left(1-e^{-\alpha_{1} t-\beta_{1} s}\right)\right\}, \quad t, s, \alpha_{0}, \alpha_{1}, \beta_{0}, \beta_{1}, \theta>0 .
$$

where $\alpha_{0}, \alpha_{1}, \beta_{0}, \beta_{1}, \theta>0$. It is clear that when $\theta \rightarrow 0$, the survival function of $(T, S)$ concludes the independence case. The model in (2.4) is a bivariate version of negative Gompertz-Makeham distribution in (1.1) and we denoted it by $\operatorname{BNGM}\left(\alpha_{0}, \alpha_{1}, \beta_{0}, \beta_{1}, \theta\right)$. Clearly, in order to decrease marginal parameters in the model and to gain a bivariate model similar to univariate negative Makeham-Gompertz, the assumption $\theta_{1}, \theta_{2} \rightarrow 0$ is asserted for the bivariate Poisson probability generating function in (2.2).

The density function of $(2.4)$ is

$$
f_{T, S}(t, s)=\left[\theta \alpha_{1} \beta_{1} e^{-\alpha_{1} t-\beta_{1} s}+\left(\beta_{0}+\theta \beta_{1} e^{-\alpha_{1} t-\beta_{1} s}\right)\left(\alpha_{0}+\theta \alpha_{1} e^{-\alpha_{1} t-\beta_{1} s}\right)\right] \bar{F}_{T, S}(t, s),
$$

and the corresponding distribution function is given by

$$
\begin{aligned}
F(t, s)= & 1-\exp \left\{-\alpha_{0} t+\theta\left(e^{-\alpha_{1} t}-1\right)\right\}-\exp \left\{-\beta_{0} s+\theta\left(e^{-\beta_{1} s}-1\right)\right\} \\
& +\exp \left\{-\alpha_{0} t-\beta_{0} s+\theta\left(e^{-\alpha_{1} t-\beta_{1} s}-1\right)\right\} .
\end{aligned}
$$

Let $\psi_{1}(t)=t^{\alpha_{0}} \exp \left\{\theta\left(t^{\alpha_{1}}-1\right)\right\}$ and $\psi_{2}(s)=s^{\beta_{0}} \exp \left\{\theta\left(s^{\beta_{1}}-1\right)\right\}$. Then $\bar{F}_{T}(t)=\psi_{1}\left(e^{-t}\right)$ and $\bar{F}_{S}(s)=\psi_{2}\left(e^{-s}\right)$ and therefore $\bar{F}_{T}^{-1}(t)=-\operatorname{Ln}\left[\psi_{1}^{-1}(t)\right]$ and $\bar{F}_{S}^{-1}(s)=-\operatorname{Ln}\left[\psi_{2}^{-1}(s)\right]$. Based on Sklar theorem, its corresponding survival copula denoted by $\operatorname{CBNGM}\left(\alpha_{0}, \alpha_{1}, \beta_{0}, \beta_{1}, \theta\right)$ is obtained by

$$
\hat{C}(t, s)=\left[\psi_{1}^{-1}(t)\right]^{\alpha_{0}}\left[\psi_{2}^{-1}(s)\right]^{\beta_{0}} \exp \left\{-\theta\left(1-\left[\psi_{1}^{-1}(t)\right]^{\alpha_{1}}\left[\psi_{2}^{-1}(s)\right]^{\beta_{1}}\right)\right\} .
$$

It is evident that $\psi($.$) is an increasing and continuous function with \psi(0)=0$ and $\psi(1)=1$, so it's a distortion function and $\hat{C}$ is a distortion copula. 


\section{Dependence Properties}

For understanding the functional relationship between random variables we have to find the corresponding dependence structure. One of the dependence notions is RCSI which implies positive dependence structure. Based on the definition, It is evident that $R C S I$ is equivalent to $\bar{F}(x, y)$ being totally positive of order 2 and $\bar{F}(x, y)$ is TP2 iff $\frac{\partial^{2} L n \bar{F}(x, y)}{\partial x \partial y}>0$ (Nelsen 2007). Thus we have the following statement.

Proposition 3.1. Let $(T, S) \sim B N G M\left(\alpha_{0}, \alpha_{1}, \beta_{0}, \beta_{1}, \theta\right)$. Then $(T, S)$ is RCSI.

Proof. For the joint vector $(T, S)$ distributed from $(2.4)$, we have

$$
\frac{\partial^{2} \operatorname{Ln} \bar{F}(t, s)}{\partial t \partial s}=\theta \alpha_{1} \beta_{1} e^{-\alpha_{1} t} e^{-\beta_{1} s}>0,
$$

which is equivalent to $\bar{F}(t, s)$ being TP2. So, $(T, S)$ is RCSI.

Since, the joint random vector distributed from BNGM model is RCSI it concludes notions such as positively quadrant dependence which indicates positive dependence structure (Nelsen (2007)). In the following, we present two local association measures which are useful in constructing independence tests for the BNGM model.

- (Clayton (1978) and Oakes (1989)) For any joint random variable $(X, Y)$ ClaytonOakes measure of dependence is

$$
\Theta(x, y)=\frac{\bar{F}(x, y) D_{12} \bar{F}(x, y)}{D_{1} \bar{F}(x, y) D_{2} \bar{F}(x, y)},
$$

where $D_{12} \bar{F}(x, y)=\frac{\partial^{2} \bar{F}(x, y)}{\partial x \partial y}, D_{1} \bar{F}(x, y)=\frac{\partial \bar{F}(x, y)}{\partial x}$ and $D_{2} \bar{F}(x, y)=\frac{\partial \bar{F}(x, y)}{\partial y}$. Clearly, if $\Theta(x, y)=1$, we will have the independence of $(X, Y)$. Moreover, if $\Theta(x, y)>(<) 1$, we will have positive (negative) dependence between $(X, Y)$. The reason for applying this measure was to check the association between the longevity of fathers and their sons based on this measure.

- (Anderson et al. (1992)) For any joint random variable $(X, Y)$, Anderson measure is defined as

$$
\Psi(x, y)=\frac{\bar{F}(x, y)}{\bar{F}(x) \bar{F}(y)} .
$$

Clearly, $\Psi(x, y)=1$ implies independence of $(X, Y)$ and $\Psi(x, y)>(<) 1$ concludes positive (negative) quadrant dependence. 
Based on the given definitions, we now present both association measures for the BNGM model.

Proposition 3.2. Let $(T, S) \sim B N G M$. Then, we have $\Theta(t, s)>1$ and $\Psi(t, s)>1$ which implies $(T, S)$ have positive dependence structure.

Proof. For any $t, s \in(0, \infty)$, we have

$$
\begin{aligned}
& \Theta(t, s)=\frac{\theta \alpha_{1} \beta_{1} e^{-\alpha_{1} t-\beta_{1} s}+\left(\beta_{0}+\theta \beta_{1} e^{-\alpha_{1} t-\beta_{1} s}\right)\left(\alpha_{0}+\theta \alpha_{1} e^{-\alpha_{1} t-\beta_{1} s}\right)}{\left(\alpha_{0}+\alpha_{1} \theta e^{-\alpha_{1} t-\beta_{1} s}\right)\left(\beta_{0}+\beta_{1} \theta e^{-\alpha_{1} t-\beta_{1} s}\right)}, \\
& \Psi(t, s)=\exp \left\{\theta\left(1-e^{-\alpha_{1} t}\right)\left(1-e^{-\beta_{1} s}\right)\right\} .
\end{aligned}
$$

Both $\Theta(t, s)$ and $\Psi(t, s)$ are greater than one, which implies $(T, S)$ have positive dependence structure.

$\Theta-1$ values

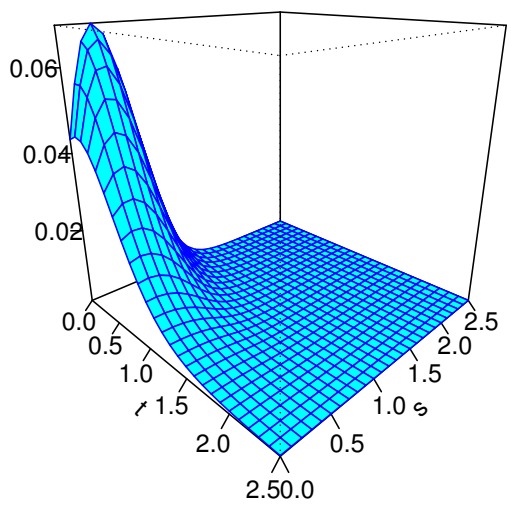

$\Psi$ values

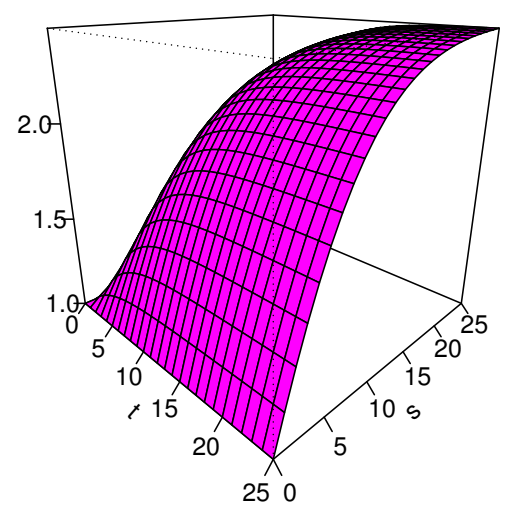

Figure 1: (Left) $\Theta_{\theta}(t, s)-1$ for $\operatorname{BNGM}(7,3,2,6,5)$ (Right) Anderson measure for parameters $\alpha_{1}=0.2, \beta_{1}=0.3, \theta=0.9$.

Based on Proposition 3.2, both measures $\Theta$ and $\Psi$, can be used to make independence tests for $(T, S)$. Also, regarding to $\Psi(x, y)$, it is clear that $\theta=0$ implies independence of $T$ and $S$ and if $\theta>0$, then we have positive dependence between $T$ and $S$. Figure 1 
shows the surface of $\Theta_{\theta}(t, s)-1$ is positive and the surface of $\Psi$ is more than 1 for the model in (2.4) concluding that the model in (2.4) has positive dependence structure.

Two famous measures of association are Kendall's tau and Spearman's rho which measure the dependence in the center of bivariate data. For the BNGM model, the direct computation of these measures are not easily tractable. For this reason, we plotted these measures against the dependence parameter $\theta$ in Figure 2. According to the figures it is clear that the values of $\rho$ and $\tau$ are positive and the BNGM distribution supports weak and intermediate dependence.
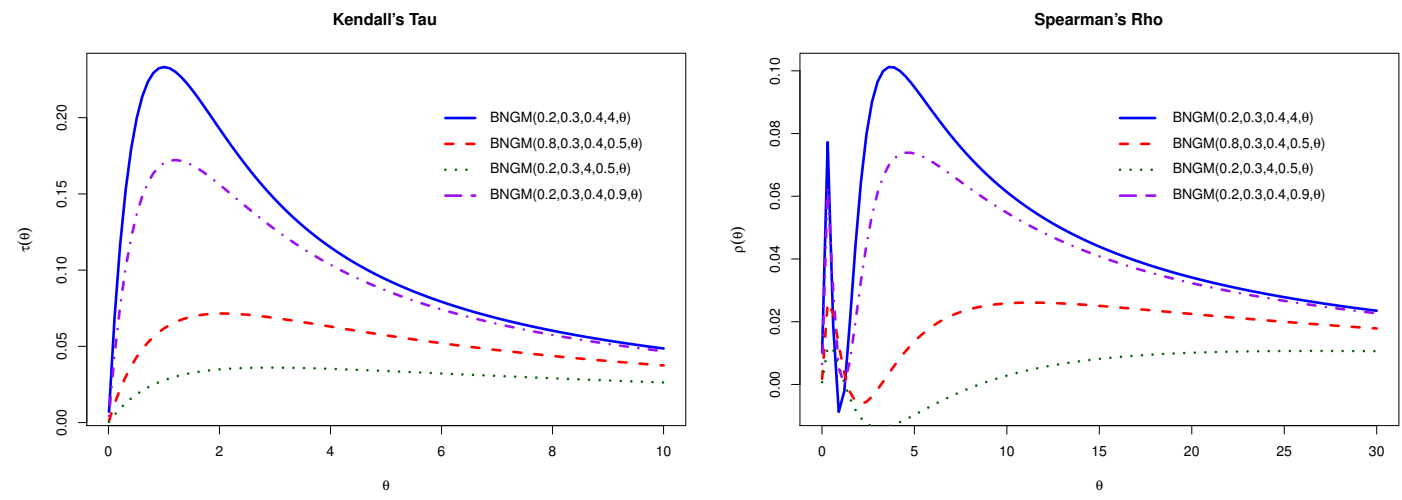

Figure 2: (Left) Kendall's $\tau$ and (Right) Spearman's $\rho$ against different dependence parameter $\theta$.

The lower and upper tail dependence coefficients $\lambda_{L}$ and $\lambda_{U}$ measure the dependence in the tails of bivariate data (Nelsen (2007)). The following statement provides the value of these measures.

Proposition 3.3. Let $(T, S) \sim B N G M\left(\alpha_{0}, \alpha_{1}, \beta_{0}, \beta_{1}, \theta\right)$. Then $\lambda_{L}=\lambda_{U}=0$.

Proof. Let $\psi_{1}(t)=t^{\alpha_{0}} \exp \left\{\theta\left(t^{\alpha_{1}}-1\right)\right\}, \psi_{2}(s)=s^{\beta_{0}} \exp \left\{\theta\left(s^{\beta_{1}}-1\right)\right\}, \phi_{i}(x)=\psi_{i}^{-1}(1-x), i=1,2$. So, $F_{T}^{-1}(t)=\bar{F}_{T}^{-1}(1-t)=-\operatorname{Ln}\left[\psi_{1}^{-1}(1-t)\right]$ and $F_{S}^{-1}(s)=\bar{F}_{S}^{-1}(1-s)=-\operatorname{Ln}\left[\psi_{2}^{-1}(1-s)\right]$. For 
every $\alpha_{0}, \alpha_{1}, \beta_{0}, \beta_{1}, \theta>0$, we have

$$
\begin{aligned}
\lambda_{L} & =\lim _{t \rightarrow 0^{+}} \frac{F\left(F_{T}^{-1}(t), F_{S}^{-1}(t)\right)}{t}, \\
& =\lim _{t \rightarrow 0^{+}} \frac{F\left(-\operatorname{Ln}\left[\psi_{1}^{-1}(1-t)\right],-\operatorname{Ln}\left[\psi_{2}^{-1}(1-t)\right]\right)}{t}, \\
& =\lim _{t \rightarrow 0^{+}} \frac{1}{t}\left[1-2 t+t^{2} \exp \left\{\theta\left(\phi_{1}^{\alpha_{1}}(t)-1\right)\left(\phi_{2}^{\beta_{1}}(t)-1\right)\right\}\right] \\
& =0 .
\end{aligned}
$$

Also,

$$
\begin{aligned}
\lambda_{U} & =\lim _{t \rightarrow 1^{-}} \frac{\bar{F}\left(F_{T}^{-1}(t), F_{S}^{-1}(t)\right)}{1-t}, \\
& =\lim _{t \rightarrow 1^{-}} \frac{\bar{F}\left(-\operatorname{Ln}\left[\psi_{1}^{-1}(1-t)\right],-\operatorname{Ln}\left[\psi_{2}^{-1}(1-t)\right]\right)}{1-t}, \\
& =\lim _{t \rightarrow 1^{-}}(1-t) \exp \left\{\theta\left(\phi_{1}^{\alpha_{1}}(t)-1\right)\left(\phi_{2}^{\beta_{1}}(t)-1\right)\right\}, \\
& =0 .
\end{aligned}
$$

Thus, the proof is completed.

Consider two random vectors $\left(X_{1}, Y_{1}\right)$ and $\left(X_{2}, Y_{2}\right)$. They can be compared in terms of their dependence structure via the upper orthant (UO) order. For every two vectors such as $\left(X_{1}, Y_{1}\right),\left(X_{2}, Y_{2}\right)$, we say $\left(X_{1}, Y_{1}\right)$ is smaller than $\left(X_{2}, Y_{2}\right)$ in upper orthant order and write $\left(X_{1}, Y_{1}\right) \prec_{U O}\left(X_{2}, Y_{2}\right)$ whenever $\bar{F}_{X_{1}, Y_{1}}(t, s) \leq \bar{F}_{X_{2}, Y_{2}}(t, s)$ for all $t, s \in R$. On noting that within the Frechet class, correlation order, upper orthant order and lower orthant order conclude each other in the bivariate case (Denuit et al 2006, pp 288). Based on the given definition, the following statement is given.

Proposition 3.4. Let $\eta=\left(\alpha_{0}, \alpha_{1}, \beta_{0}, \beta_{1}\right),(T, S) \sim B N M G(\eta, \theta)$ and $\left(T^{\prime}, S^{\prime}\right) \sim B N M G\left(\eta, \theta^{\prime}\right)$. For any fixed value $\eta$ if $\theta<\theta^{\prime}$ then $\left(T^{\prime}, S^{\prime}\right) \prec_{U O}(T, S)$.

Proof. For any $t, s \in R$ and $\theta<\theta^{\prime}$, we have

$$
\begin{aligned}
\bar{F}_{\theta^{\prime}}(t, s) & =\exp \left\{-\alpha_{0} t-\beta_{0} s-\theta^{\prime}\left(1-e^{-\alpha_{1} t-\beta_{1} s}\right)\right\} \\
& \leq \exp \left\{-\alpha_{0} t-\beta_{0} s-\theta\left(1-e^{-\alpha_{1} t-\beta_{1} s}\right)\right\} \\
& \leq \bar{F}_{\theta}(t, s) .
\end{aligned}
$$

So, the desired result is concluded. 


\section{Hazard Functions}

One of the main reliability concepts is studying the behaviour of univariate and bivariate hazard rates corresponding to $(T, S)$. In the NGM model given in (1.2), $T$ and $S$ have decreasing hazard rates (Marshall and Olkin (2007)). The conditional survival function of $T \mid S>s$ and $T \mid S=s$ based on BNGM model are

$$
\bar{F}_{T \mid S>s}(t \mid s)=\exp \left\{-\alpha_{0} t+\theta e^{-\beta_{1} s}\left(e^{-\alpha_{1} t}-1\right)\right\},
$$

and

$$
\bar{F}_{T \mid S=s}(t \mid s)=\frac{\frac{\partial \bar{F}(t, s)}{\partial s}}{\frac{\partial \bar{F}(s)}{\partial s}}=\frac{\beta_{0}+\beta_{1} e^{-\beta_{1} s} \theta e^{-\alpha_{1} t}}{\beta_{0}+\beta_{1} \theta e^{-\beta_{1} s}} \bar{F}_{T \mid S>s}(t \mid s) .
$$

respectively. So, the hazard function of $T \mid S>s$ and $T \mid S=s$ are

$$
h_{T \mid S>s}(t)=\alpha_{0}+\alpha_{1} \theta e^{-\alpha_{1} t-\beta_{1} s}
$$

and

$$
h_{T \mid S=s}(t)=h_{T \mid S>S}(t)+\frac{\alpha_{1} \beta_{1} \theta e^{-\alpha_{1} t-\beta_{1} S}}{\beta_{0}+\beta_{1} \theta e^{-\alpha_{1} t-\beta_{1} S}},
$$

consequently, where $\alpha_{0}, \alpha_{1}, \beta_{0}, \beta_{1}, \theta, t, s>0$.

The next proposition gives the monotonicity behaviour of the hazard function for $T \mid S>s$ and $T \mid S=s$.

Proposition 4.1. Let $(T, S) \sim B N G M\left(\alpha_{0}, \alpha_{1}, \beta_{0}, \beta_{1}, \theta\right)$. Then, the conditionals $T \mid S>s$, $S|T>t, T| S=s$ and $S \mid T=t$ have decreasing failure rates.

Proof. Since $T \mid S>s \sim N G M\left(\alpha_{0}, \alpha_{1}, \theta e^{-\beta_{1} s}\right)$ and $S \mid T>t \sim N G M\left(\beta_{0}, \beta_{1}, \theta e^{-\alpha_{1} t}\right)$, based on Marshall and Olkin (2007), the NGM model has decreasing failure rate and hence both $T \mid S>s$ and $S \mid T>t$ have decreasing failure rates. For $T \mid S=s$, we have

$$
h_{T \mid S=S}^{\prime}(t \mid s)=h_{T \mid S>S}^{\prime}(t \mid s)-\frac{\alpha_{1}^{2} \beta_{0} \beta_{1} \theta e^{-\alpha_{1} t-\beta_{1} s}}{\left(\beta_{0}+\beta_{1} \theta e^{-\alpha_{1} t-\beta_{1} s}\right)^{2}}<0,
$$

and so $T \mid S=s$ has decreasing failure rate. The result for $S \mid T=t$ can be derived similarly. 

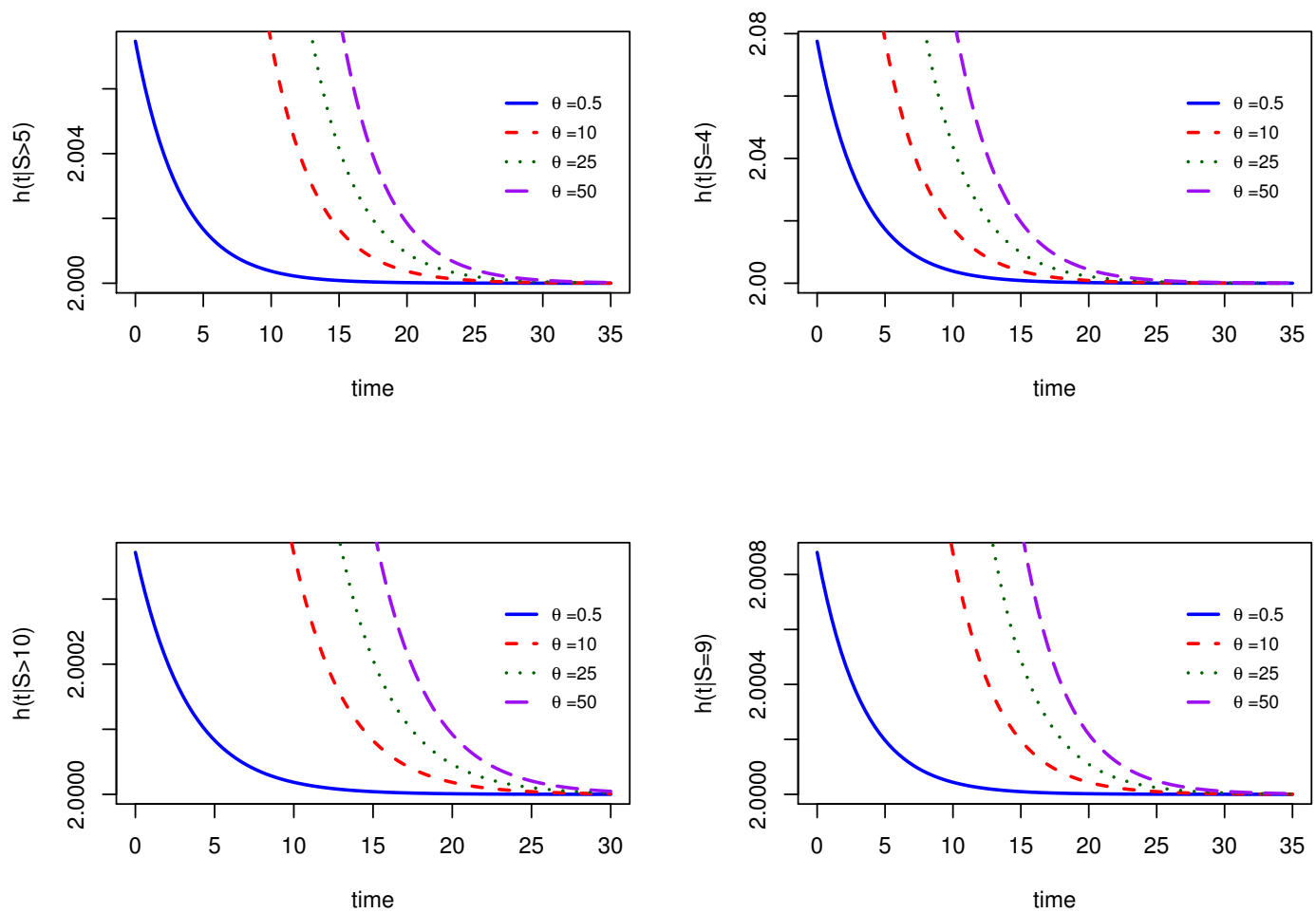

Figure 3: Plots of $h_{T \mid S>S}$ (left side) and $h_{T \mid S=s}$ (right side) of $\operatorname{BNGM}(2,0.3,2,0.6, \theta)$ for $\theta=0.5,10,25,50$.

For seeing the behaviour of hazard functions in proposition 4.1, some plots have been obtained. Figure 3 shows that both hazard conditionals $h_{T \mid S>S}$ and $h_{T \mid S=s}$ have decreasing rate. Clearly, the dependence parameter $\theta$ increases the hazard function. Also the conditional hazard functions have decreasing rate.

For the bivariate case, Johnson and Kotz (1975) introduced the hazard gradient vector $\left(h_{1}(x, y), h_{2}(x, y)\right)$ where $h_{1}(x, y)$ is the hazard rate of the conditional distribution of $X$ given $Y>y$ and $h_{2}(x, y)$ is the hazard rate of $Y \mid X>x$. Also, it can be shown that $h_{1}(x, y)=-\frac{\partial}{\partial x} \operatorname{Ln} \bar{F}(x, y)$ and $h_{2}(x, y)=-\frac{\partial}{\partial y} \operatorname{Ln} \bar{F}(x, y)$. Based on their idea, bivariate increasing (decreasing) failure rate is concluded for all $(x, y)$ when the function $h_{1}$ is an increasing (decreasing) function of $x$ and $h_{2}$ is an increasing (decreasing) function of $y$. 
Proposition 4.2. Let $(T, S) \sim B N G M$. Then the BNGM model has decreasing failure rate.

Proof. From (2.4), we get $\frac{\partial}{\partial t} h_{1}(t, s)=-\alpha_{1}^{2} \theta e^{-\alpha_{1} t-\beta_{1} s}<0$ and $h_{2}(t, s)=-\beta_{1}^{2} \theta e^{-\alpha_{1} t-\beta_{1} s}<0$ and this implies that $\bar{F}_{T, S}$ in (2.4) has bivariate decreasing failure rate.

\section{Ageing Concepts}

One of the main aspects in reliability theory is "ageing" which is an inherent property of a unit that may be a system of components or even a living organism. Some notable measures of ageing are failure rate, mean residual life, etc. Based on Szymkowiak (2020), for any univariate absolutely continuous random variable $X$ with survival function $\bar{F}$ and density function $f$, the univariate hazard function at time $t$ is defined as $h_{X}(t)=\frac{f(t)}{\bar{F}(t)}$. The notion $h_{X}(t)$ can be interpreted as the local infinitesimal conditional probability of an instantaneous failure occurring immediately after the time point $t$ given that the unit has survived until $t$. Jiang et al. (2003) states that the representation of ageing of a system by failure rate is qualitative. In order to evaluate the ageing property of a system quantitatively they developed a new notion, called ageing intensity (AI). The AI for univariate absolutely continuous random variable $X$ at time $t$, denoted by $L_{X}(t)$, is defined as

$$
L_{X}(t)=\frac{h_{X}(t)}{H_{X}(t)}
$$

where $h_{X}(t)$ is the hazard function and $H_{X}(t)=\frac{1}{t} \int_{0}^{t} h_{X}(x) d x$ is the failure rate average. The larger the ageing intensity, the stronger the tendency of ageing. For the model in 1.1 univariate $\mathrm{AI}$ is

$$
L_{X}(t)=\frac{\delta t+\lambda \theta e^{-\lambda t}}{\delta t+\theta\left(1-e^{-\lambda t}\right)} .
$$

Figure 4 illustrated the behaviour of univariate AI.

Based on Figure 4, tendency of ageing for $\operatorname{NGM}(\delta, \lambda, \theta)$ decreases as time passes for different values of $\delta, \lambda, \theta$.

For bivariate absolutely continuous random vector $(X, Y)$ with survival function $\bar{F}$, the gradient of hazard functions is defined as $\mathbf{h}_{X, Y}(x, y)=\left(h_{1}(x, y), h_{2}(x, y)\right)$, where $h_{1}(x, y)=-\frac{\partial}{\partial x} \ln \bar{F}(x, y)$ and $h_{2}(x, y)=-\frac{\partial}{\partial y} \ln \bar{F}(x, y)$. Consequently, the vector of the failure rate averages is defined as $\mathbf{H}_{X, Y}(x, y)=\left(H_{1}(x, y), H_{2}(x, y)\right)$ where $H_{1}(x, y)=$ $\frac{1}{x} \int_{0}^{x} h_{1}(u, y) d u$ and $H_{2}(x, y)=\frac{1}{y} \int_{0}^{y} h_{2}(x, v) d v$. Based on the preceding notions we define the vector of ageing intensities as following: 


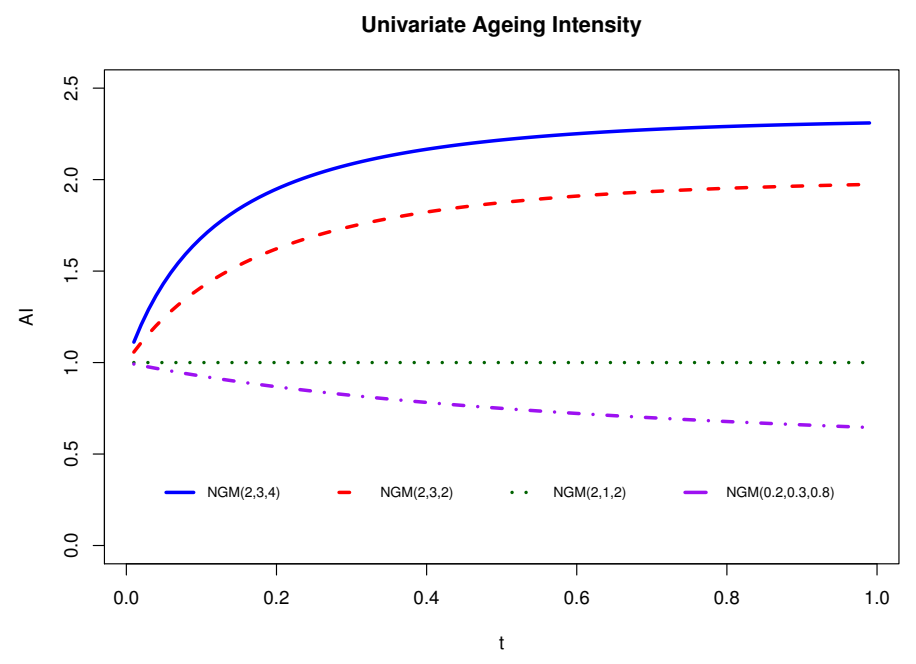

Figure 4: Ageing intensity of $\operatorname{NGM}(\delta, \lambda, \theta)$.

Definition 5.1. Let $(X, Y)$ be a bivariate absolutely continuous random vector with survival function $\bar{F}$, gradient of hazard functions $\mathbf{h}_{X, Y}$ and vector of the failure rate averages $\mathbf{H}_{X, Y}$. The vector of ageing intensities for absolutely continuous random vector $(X, Y)$ (denoted by $B A I(X, Y))$ is defined as $\mathbf{L}_{X, Y}(x, y)=\left(L_{1}(x, y), L_{2}(x, y)\right)$, where $L_{i}(x, y)=\frac{h_{i}(x, y)}{H_{i}(x, y)}$ for $i=1,2$.

According to the given definition we now derive the ageing intensity for the BNGM model.

Proposition 5.1. Let $(T, S) \sim B N G M\left(\alpha_{0}, \alpha_{1}, \beta_{0}, \beta_{1}, \theta\right)$. Then, the bivariate ageing intensity vector of $(T, S)$ is

- $L_{1}(t, s)=\frac{\left(\alpha_{0} t+\alpha_{1} \theta t S\left(t, \alpha_{1}\right) S\left(s, \beta_{1}\right)\right)}{\left(\alpha_{0} t+\theta S\left(s, \beta_{1}\right)\left(1-S\left(x, \alpha_{1}\right)\right)\right)^{\prime}}$

- $L_{2}(t, s)=\frac{\left(\beta_{0} s+\beta_{1} \theta s S\left(t, \alpha_{1}\right) S\left(s, \beta_{1}\right)\right)}{\left(\beta_{0} s+\theta S\left(t, \alpha_{1}\right)\left(1-S\left(y, \beta_{1}\right)\right)\right)}$,

where $S(x, \gamma)=\exp (-\gamma x)$ for all $\gamma, x>0$.

Proof. For any $\alpha_{0}, \alpha_{1}, \beta_{0}, \beta_{1}, \theta>0$, we have

$$
h_{1}(t, s)=\alpha_{0}+\alpha_{1} \theta e^{-\alpha_{1} t-\beta_{1} s},
$$


and

$$
h_{2}(t, s)=\beta_{0}+\beta_{1} \theta e^{-\alpha_{1} t-\beta_{1} s} .
$$

Thus, we have

$$
\begin{aligned}
R_{1}(t, s) & =\frac{1}{t} \int_{0}^{t} r_{1}(x, s) d x \\
& =\frac{1}{t} \int_{0}^{t} \alpha_{0}+\alpha_{1} \theta e^{-\alpha_{1} x-\beta_{1} s} d x \\
& =\alpha_{0}+\theta e^{-\beta_{1} s} \cdot \frac{1-e^{-\alpha_{1} t}}{t},
\end{aligned}
$$

and similarly $R_{2}(t, s)=\beta_{0}+\theta e^{-\alpha_{1} t} \cdot \frac{1-e^{-\beta_{1} s}}{s}$. Based on the fact that $\left(B A I_{1}(T, S), B A I_{2}(T, S)\right)=$ $\left(\frac{r_{1}(t, s)}{R_{1}(t, s)}, \frac{r_{2}(t, s)}{R_{2}(t, s)}\right)$ we derive the given statement.

Based on Figure 5, we have plotted the perspective and contour plot for $B A I_{1}(T, S ; \theta=$ 1) $-B A I_{1}(T, S ; \theta=0.5)$ and $B A I_{2}(T, S ; \theta=1)-B A I_{2}(T, S ; \theta=0.5)$ with fixed parameter $\left(\alpha_{0}, \alpha_{1}, \beta_{0}, \beta_{1}\right)=(5,3,5,4)$. The figures indicate that both the plots are positive and this shows that $B A I_{1}$ and $B A I_{2}$ are both increasing w.r.t. $\theta$ within the range of the dependence parameter $\theta \in(0.5,1)$.

Investigating ageing concepts for reliability distributions is of great importance. The notion of having the lack of memory property is given in Lai and Xie (2006). In a twocomponent system, lack of memory property states that the conditional probability of two components surviving to times $(x+t, y+t)$ given surviving to times $(t, t)$ is equal to the unconditional probability of these two components surviving to times $(x, y)$. The bivariate Marshall-Olkin exponential distribution is known for its extension of exponential distribution and it's the only bivariate distribution with exponential marginals satisfying the bivariate lack of memory property. However, other bivariate distributions don't satisfy the lack of memory property and the notions BNWU-I (BNBU-I) and BNWU-II (BNBU-II) are defined. This notion can be interpreted as the conditional survival probability of two components of different ages being more (less) than the corresponding survival probability $\bar{H}$ of two new components. The following corollary gives the relation of BNWU-I and BNWU-II and presents the corresponding univariate concept.

Corollary 5.1. Based on the notions BNWU-I (BNBU-I) and BNWU-II (BNBU-II), we have

- If $t_{1}=t_{2}=t$, then BNWU-I and BNWU-II are equal. 
- If $y, t_{2} \rightarrow 0$, then $\bar{F}_{T}\left(x+t_{1}\right) \geq \bar{F}_{T}(x) \bar{F}_{T}\left(t_{1}\right)$ that implies the notion new worse than used (NWU) for random variable $X$.

- If $x, t_{1} \rightarrow 0$, then $\bar{F}_{S}\left(y+t_{2}\right) \geq \bar{F}_{S}(y) \bar{F}_{S}\left(t_{2}\right)$ that implies the notion NWU for random variable $Y$.
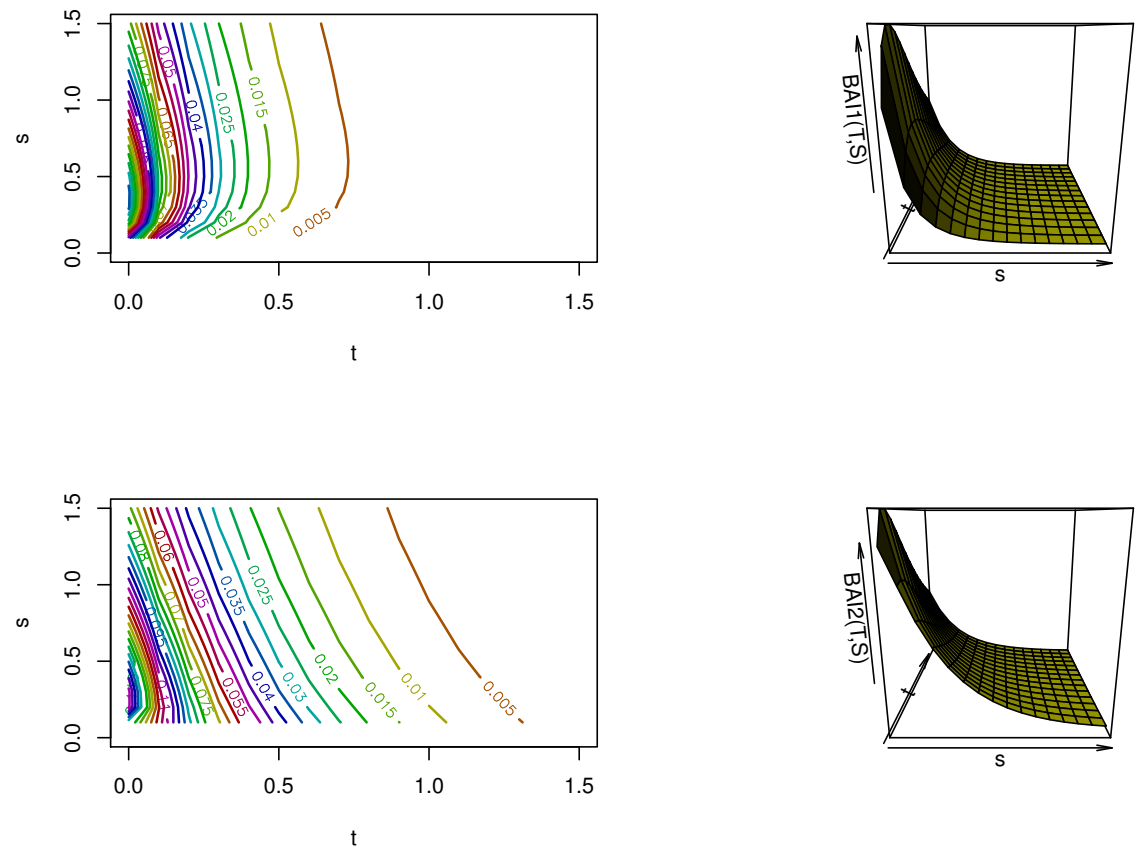

Figure 5: (First row) On the left, contour plot and on the right perspective plot for $B A I_{1}(T, S ; \theta=1)-B A I_{1}(T, S ; \theta=0.5)$ when $\left(\alpha_{0}, \alpha_{1}, \beta_{0}, \beta_{1}\right)=(5,3,5,4)$. (Second row) On the left, contour plot and on the right perspective plot for $B A I_{2}(T, S ; \theta=1)$ $B A I_{2}(T, S ; \theta=0.5)$ when $\left(\alpha_{0}, \alpha_{1}, \beta_{0}, \beta_{1}\right)=(5,3,5,4)$.

Based on the given definition, we present some ageing concepts for the BNGM model.

Proposition 5.2. Let $(T, S) \sim B N G M\left(\alpha_{0}, \alpha_{1}, \beta_{0}, \beta_{1}, \theta\right)$. Then

- $(T, S)$ satisfies BNWU-I, 
- $(T, S)$ satisfies BNWU-II,

- $T$ is new worse than used (NWU),

- $S$ is NWU.

Proof. For all $x, y, t_{1}, t_{2}>0$, we have

$$
\begin{aligned}
\frac{\bar{F}_{T, S}\left(x+t_{1}, y+t_{2}\right)}{\bar{F}_{T, S}(x, y)}= & \exp \left\{-\alpha_{0} t_{1}-\beta_{0} t_{2}-\theta\left(1-e^{-\alpha_{1} t_{1}-\beta_{1} t_{2}}\right)\right\}, \\
& \times \exp \left\{\theta\left[1-e^{-\alpha_{1} t_{1}-\beta_{1} t_{2}}+\theta e^{-\alpha_{1}\left(x+t_{1}\right)-\beta_{1}\left(y+t_{2}\right)}\right]\right\}, \\
= & \bar{F}_{T, S}\left(t_{1}, t_{2}\right) \cdot \exp \left\{\theta\left(1-e^{-\alpha_{1} t_{1}-\beta_{1} t_{2}}\right)\left(1-e^{-\alpha_{1} x-\beta_{1} y}\right)+\theta e^{-\alpha_{1} x-\beta_{1} y}\right\}, \\
\geq & \bar{F}_{T, S}\left(t_{1}, t_{2}\right) .
\end{aligned}
$$

Thus, we have BNWU-II and if we set $t_{1}=t_{2}=t$ we get BNWU-I. Using the definition of BNWU-I (BNBU-I) and BNWU-II (BNBU-II), we can obtain the third and fourth statement. So, the proof is complete.

\section{Stress-Strength Based on Competing Risks}

In the reliability literature, stress-strength model is marked out as an analysis for a reliability system in terms of random variables $X$ indicating stress (supply) exposed to the system and $Y$ as the strength (demand) of the system available to bear the stress. The system breaks down whenever the stress surpasses the strength. So, the index $R=P(X<Y)$ is the reliability considering the failure mode described by the stressstrength relation. The stress-strength index can be computed in terms of competing risk given in Shih and Emura (2019). Based on competing risk models, failure times $X$ and $Y$ are called latent failure times. According to the failure time $R=\min (X, Y)$ and failure cause $C=1$ if $X<Y$ or $C=2$ if $X>Y$, we give the sub-distribution functions as

$$
F^{*}(1, r)=P(C=1, T \leq r)=\int_{0}^{r} f^{*}(1, z) d z,
$$

and

$$
F^{*}(2, r)=P(C=2, T \leq r)=\int_{0}^{r} f^{*}(2, z) d z,
$$

where $f^{*}(1, t)=-\partial \bar{F}(x, y) /\left.\partial x\right|_{x=y=t}$ and $f^{*}(2, t)=-\partial \bar{F}(x, y) /\left.\partial y\right|_{x=y=t}$ that are called subdensity functions. Then the stress-strength index is given by $P(X<Y)=F^{*}(1, \infty)$ and $P(X>Y)=F^{*}(2, \infty)$. First we will present the following lemma that will help us in finding the stress-strength index. 
Lemma 6.1. Let $\omega(\gamma, \theta, b)=\int_{0}^{\infty} \exp \left\{-\gamma t+\theta e^{-b t}-\theta\right\} d t$. Then

$$
\omega(\gamma, \theta, b)=\frac{1}{b} E\left(\frac{b}{\gamma+b N}\right),
$$

where $N$ is a Poisson random variable with parameter $\theta$.

Proof. Using the transformation $w=e^{-b t}$, we get

$$
\omega(\gamma, \theta, b)=\frac{e^{-\theta}}{b} \int_{0}^{1} w^{\gamma / b-1} e^{\theta w} d w .
$$

Based on the Maclaurin series we have

$$
\begin{aligned}
\omega(\gamma, \theta, b) & =\frac{e^{-\theta}}{b} \int_{0}^{1} w^{\gamma / b-1} \sum_{n=0}^{\infty} \frac{(\theta w)^{n}}{n !} d w, \\
& =\frac{e^{-\theta}}{b} \sum_{n=0}^{\infty} \frac{\theta^{n}}{n !} \frac{1}{\gamma / b+n} \\
& =\frac{1}{b} E\left(\frac{b}{\gamma+b N}\right) .
\end{aligned}
$$

So, the proof is complete.

Based on obtaining the reliability of an equipment or the survival of a living object, it is crucial to consider the stress conditions of the functioning environment. That is, uncertainty about the main surrounding stress to be encountered should considered as random. In the simplest stress-strength model, the component (or living object) $X$ is the stress placed on the unit by the operating environment and the component (or living object) $Y$ is the strength of the unit. A unit (or living object) will perform its intended function if the strength is greater than the stress imposed upon it. Then, reliability would be defined as the probability that the unit (or living object) has the strength to overcome the stress. On the basis of competing risk model, stress-strength index for the BNGM model is given in the proceeding statement.

Proposition 6.1. If $(T, S) \sim B N G M\left(\alpha_{0}, \alpha_{1}, \beta_{0}, \beta_{1}, \theta\right)$, then

$$
P(S<T)=\beta_{0} \omega\left(\alpha_{0}+\beta_{0}, \theta, \alpha_{1}+\beta_{1}\right)+\beta_{1} \theta \omega\left(\alpha_{0}+\beta_{0}+\alpha_{1}+\beta_{1}, \theta, \alpha_{1}+\beta_{1}\right),
$$

where $\omega(\gamma, \theta, b)=\frac{1}{b} E\left(\frac{b}{\gamma+b N}\right)$ such that $N \sim P(\theta)$. In particular, as $\theta \rightarrow 0$, then $P(S<T)=$ $\frac{\beta_{0}}{\alpha_{0}+\beta_{0}}$. 
Proof. Based on the proposed model (2.4), we have

$$
f^{*}(2, r)=-\left.\frac{\partial \bar{F}_{T, S}(t, s)}{\partial t}\right|_{t=s=r}=\left(\beta_{0}+\beta_{1} \theta e^{-\left(\alpha_{1}+\beta_{1}\right) r}\right) \bar{F}_{T, S}(r, r)
$$

So,

$$
F^{*}(2, r)=\int_{0}^{r}\left(\beta_{0}+\beta_{1} \theta e^{-\left(\alpha_{1}+\beta_{1}\right) x}\right) \bar{F}_{T, S}(x, x) d x
$$

Thus,

$$
\begin{aligned}
P(S<T)= & \int_{0}^{\infty}\left(\beta_{0}+\beta_{1} \theta e^{-\left(\alpha_{1}+\beta_{1}\right) x}\right) \bar{F}_{T, S}(x, x) d x \\
= & \beta_{0} \int_{0}^{\infty} \exp \left\{-\left(\alpha_{0}+\beta_{0}\right) x+\theta e^{-\left(\alpha_{1}+\beta_{1}\right) x}-\theta\right\} d x \\
& +\beta_{1} \theta \int_{0}^{\infty} \exp \left\{-\left(\alpha_{0}+\beta_{0}+\alpha_{1}+\beta_{1}\right) x+\theta e^{-\left(\alpha_{1}+\beta_{1}\right) x}-\theta\right\} d x,
\end{aligned}
$$

By plugging lemma 6.1 in (6.2) we get the first statement. For the the second statement, using (6.1) and $\theta \rightarrow 0$, we have

$$
P(S<T)=\int_{0}^{\infty} \beta_{0} \exp \left\{-\left(\alpha_{0}+\beta_{0}\right) x\right\} d x=\frac{\beta_{0}}{\alpha_{0}+\beta_{0}} .
$$

So, the proof is complete.

To illustrate the variation of the stress-strength index for $\operatorname{BNGM}\left(\alpha_{0}, \alpha_{1}, \beta_{0}, \beta_{1}, \theta\right)$ model, different curves are given in Figure 6 . The plot is the stress-strength index versus the dependence parameter with a variation in any of the four marginal parameters $\left(\alpha_{0}, \alpha_{1}, \beta_{0}, \beta_{1}\right)$. Clearly as $\theta \rightarrow 0$, the stress-strength index converges to $\frac{\beta_{0}}{\alpha_{0}+\beta_{0}}$.

\section{Estimation and Simulation}

Throughout this section, we study the behaviour of the BNGM model by presenting an algorithm to generate random data and give suitable plots to illustrate their variation. Next, estimators for the marginal and dependence parameters are given. Finally, a performance analysis for the introduced estimators are given and they are compared based on some criteria. 
Stress-Strength for $\operatorname{BNGM}\left(\alpha_{0}, 0.3,0.4,0.5, \theta\right)$

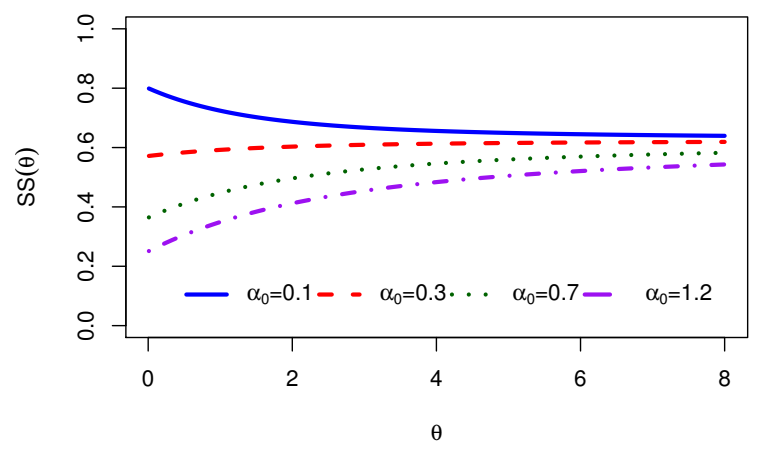

Figure 6: Stress-Strength index versus the dependence parameter $\theta$ for $\operatorname{BNGM}\left(\alpha_{0}, \alpha_{1}, \beta_{0}, \beta_{1}, \theta\right)$.

\subsection{Random Number Generation}

We can obtain samples from the BNGM model based on the construction in (2.1). So, the following algorithm is presented.

Algorithm 1 Random number generation from $\operatorname{BNGM}\left(\alpha_{0}, \alpha_{1}, \beta_{0}, \beta_{1}, \theta\right)$.

Step 1. Generate $\left(n_{1}, n_{2}\right)$ from a bivariate Poisson distribution with parameters $\left(\theta_{1}, \theta_{2}, \theta_{12}\right)$ for small values of $\theta_{1}, \theta_{2}$ such that $\theta_{1}, \theta_{2} \rightarrow 0$.

Step 2. Generate $x_{0}$ and $y_{0}$ from exponential distributions with parameters $\alpha_{0}$ and $\beta_{0}$, respectively.

Step 3. Generate $x_{1}, \ldots, x_{n_{1}}$ and $y_{1}, \ldots, y_{n_{2}}$ from exponential distributions with parameters $\alpha_{1}$ and $\beta_{1}$, respectively.

Step 4. Set $t=\min \left\{x_{0}, x_{1}, \ldots, x_{n_{1}}\right\}$ and $s=\min \left\{y_{0}, y_{1}, \ldots, y_{n_{2}}\right\}$.

Step 5. The desired pair is $(t, s)$.

Based on Algorithm 1 scatter plots and contour plots are given in Figure 7. Based on Figure 2, the dependency of the generated data first increase and then decline as $\theta$ 
increases. This can also be seen in Figure 7. Since, the generated data are of GompertzMakeham type, they are centralized near zero in each axis. Clearly, as the value of dependency decreases the generated data become more concentrated near zero.
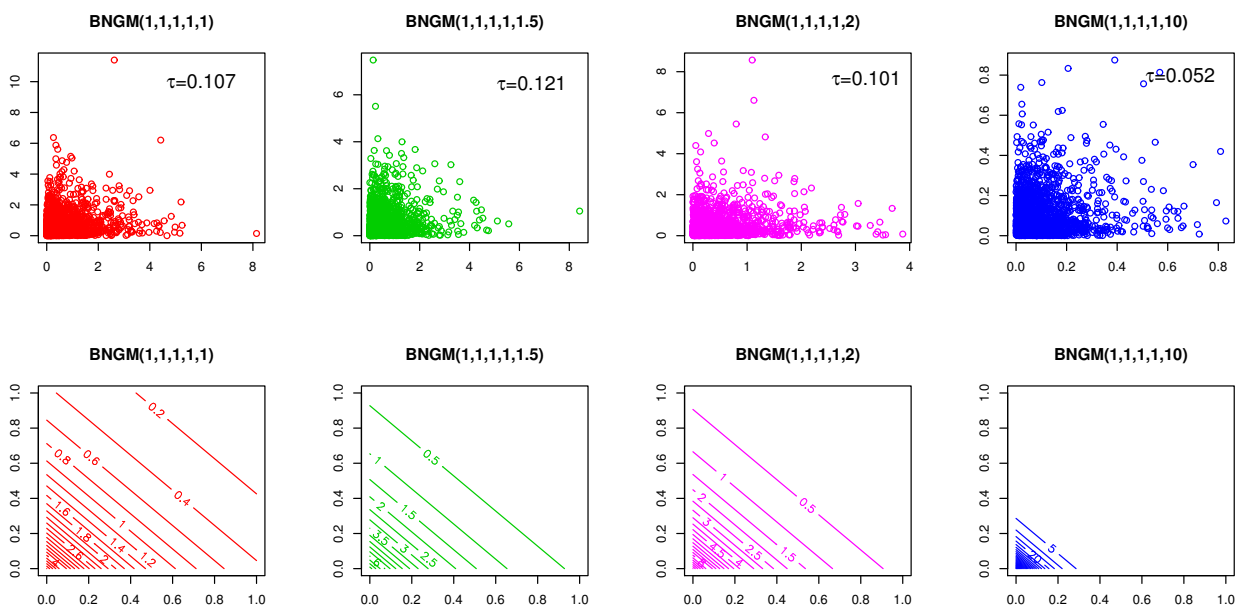

Figure 7: Scatter plots (first row) and contour plots (second row) for different values of dependence parameter $\theta$ for $\operatorname{BNGM}(1,1,1,1, \theta)$. The value of Kendall's $\tau$ is given in the scatter plots.

\subsection{Estimation and Performance Analysis}

Suppose we have a bivariate random sample $\left(T_{1}, S_{1}\right), \ldots,\left(T_{n}, S_{n}\right)$ distributed as $(T, S)$. We want to estimate the marginal parameters $\left(\alpha_{0}, \alpha_{1}, \beta_{0}, \beta_{1}\right)$ of BNGM model using maximum likelihood estimation (MLE) and estimate the dependence parameter $\theta$ using MLE, Rho-inversion and Tau-inversion methods. We will explain these these methods as following.

\subsubsection{MLE}

Suppose we have the observations $\left(t_{1}, s_{1}\right), \ldots,\left(t_{n}, s_{n}\right)$ distributed from the pdf given in (2.5). Let $\gamma=\left(\alpha_{0}, \alpha_{1}, \beta_{0}, \beta_{1}, \theta\right), g_{j}\left(\alpha_{1}, \beta_{1}\right)=\exp \left\{-\alpha t_{j}-\beta s_{j}\right\}$ and

$$
\Delta_{j}\left(\alpha_{0}, \alpha_{1}, \beta_{0}, \beta_{1}, \theta\right)=\theta \alpha_{1} g_{j}\left(\alpha_{1}, \beta_{1}\right)+\left[\left(\beta_{0}+\theta \beta_{1} g_{j}\left(\alpha_{1}, \beta_{1}\right)\right)\left(\alpha_{0}+\theta \alpha_{1} g_{j}\left(\alpha_{1}, \beta_{1}\right)\right)\right] .
$$


So, the log-likelihood function based on observations is

$$
l(\gamma)=\sum_{j=1}^{n} \log \left[\Delta_{j}\left(\alpha_{0}, \alpha_{1}, \beta_{0}, \beta_{1}, \theta\right)\right]-\alpha_{0} \sum_{j=1}^{n} t_{j}-\beta_{0} \sum_{j=1}^{n} s_{j}-n \theta+\theta \sum_{j=1}^{n} g_{j}\left(\alpha_{1}, \beta_{1}\right) .
$$

Now, we want to find MLE of $\gamma$ by maximizing (7.1). The estimates of $\gamma$ can be obtained by solving the following non-linear equations simultaneously:

$$
\begin{aligned}
\frac{\partial l(\gamma)}{\partial \alpha_{0}}= & \sum_{j=1}^{n}\left[\frac{\beta_{0}+\theta \beta_{1} g_{j}\left(\alpha_{1}, \beta_{1}\right)}{\Delta_{j}\left(\alpha_{0}, \alpha_{1}, \beta_{0}, \beta_{1}, \theta\right)}-t_{j}\right]=0, \\
\frac{\partial l(\gamma)}{\partial \beta_{0}}= & \sum_{j=1}^{n}\left[\frac{\alpha_{0}+\theta \alpha_{1} g_{j}\left(\alpha_{1}, \beta_{1}\right)}{\Delta_{j}\left(\alpha_{0}, \alpha_{1}, \beta_{0}, \beta_{1}, \theta\right)}-s_{j}\right]=0, \\
\frac{\partial l(\gamma)}{\partial \alpha_{1}}= & \sum_{j=1}^{n} \frac{g_{j}\left(\alpha_{1}, \beta_{1}\right)}{\Delta_{j}\left(\alpha_{0}, \alpha_{1}, \beta_{0}, \beta_{1}, \theta\right)}\left[\theta \beta_{1}-\theta \alpha_{1} \beta_{1} t_{j}-\theta \beta_{1} t_{j}\left(\alpha_{0}+\theta \alpha_{1} g_{j}\left(\alpha_{1}, \beta_{1}\right)\right)\right. \\
& \left.+\left(\beta_{0}+\theta \beta_{1} g_{j}\left(\alpha_{1}, \beta_{1}\right)\right)\left(\theta-\theta \alpha_{1} t_{j}\right)\right]-\theta \sum_{j=1}^{n} t_{j} g_{j}\left(\alpha_{1}, \beta_{1}\right)=0, \\
\frac{\partial l(\gamma)}{\partial \beta_{1}}= & \sum_{j=1}^{n} \frac{g_{j}\left(\alpha_{1}, \beta_{1}\right)}{\Delta_{j}\left(\alpha_{0}, \alpha_{1}, \beta_{0}, \beta_{1}, \theta\right)}\left[\theta \alpha_{1}-\theta \alpha_{1} \beta_{1} s_{j}-\theta \alpha_{1} s_{j}\left(\beta_{0}+\theta \beta_{1} g_{j}\left(\alpha_{1}, \beta_{1}\right)\right)\right. \\
& \left.+\left(\alpha_{0}+\theta \alpha_{1} g_{j}\left(\alpha_{1}, \beta_{1}\right)\right)\left(\theta-\theta \beta_{1} s_{j}\right)\right]-\theta \sum_{j=1}^{n} s_{j} g_{j}\left(\alpha_{1}, \beta_{1}\right)=0, \\
\frac{\partial l(\gamma)}{\partial \theta}= & \sum_{j=1}^{n} \frac{g_{j}\left(\alpha_{1}, \beta_{1}\right)}{\Delta_{j}\left(\alpha_{0}, \alpha_{1}, \beta_{0}, \beta_{1}, \theta\right)}\left[\alpha_{1} \beta_{1}+\beta_{1}\left(\alpha_{0}+\theta \alpha_{1} g_{j}\left(\alpha_{1}, \beta_{1}\right)\right)+\alpha_{1}\left(\beta_{0}+\theta \beta_{1} g_{j}\left(\alpha_{1}, \beta_{1}\right)\right)\right] \\
& -n+\sum_{j=1}^{n} g_{j}\left(\alpha_{1}, \beta_{1}\right)=0 .
\end{aligned}
$$

However, this method is less efficient than the direct maximization of log-likelihood. In order to maximize the log-likelihood function we use the function optim in the $\mathrm{R}$ software. Initial values for optimization are calculated based on global non-linear optimization package "Rsolnp" in R software version 3.6.1. 


\subsubsection{Rho-Inversion}

The dependence measure Spearman Rho $(\rho)$ is defined as the correlation of the ranks from joint observations. Let $\bar{F}_{\theta}$ be the bivariate survival function with dependence parameter $\theta$ and $\bar{F}_{1}, \bar{F}_{2}$ be its corresponding marginals, then the population version of Spearman's Rho is

$$
\rho_{\theta}=12 \int_{(0, \infty)^{2}}\left[\bar{F}_{\theta}(x, y)-\bar{F}_{1}(x) \bar{F}_{2}(y)\right] d \bar{F}_{1}(x) d \bar{F}_{2}(y),
$$

where $\theta$ is the dependence parameter of the population. Let $\left(R_{i}, S_{i}\right)$ be ranks corresponding to observations. Then sample version of Spearman's Rho is defined as

$$
\rho_{n}=\frac{\sum_{i=1}^{n}\left(R_{i}-\bar{R}\right)\left(S_{i}-\bar{S}\right)}{\sqrt{\sum_{i=1}^{n}\left(R_{i}-\bar{R}\right)^{2} \sum_{i=1}^{n}\left(S_{i}-\bar{S}\right)^{2}}},
$$

where $\bar{R}=\bar{S}=\frac{n+1}{2}$. Also, we can re-write the Spearman's Rho as

$$
\rho_{n}=\frac{12}{n(n-1)(n+1)} \sum_{i=1}^{n} R_{i} S_{i}-\frac{n+1}{n-1} .
$$

For the $\operatorname{BNGM}\left(\alpha_{0}, \alpha_{1}, \beta_{0}, \beta_{1}, \theta\right)$ model we estimate the marginal parameters $\left(\alpha_{0}, \alpha_{1}, \beta_{0}, \beta_{1}\right)$ using MLE and then estimate dependence parameter $\theta$ based on Rho-inversion method. To estimate $\theta$, first, we calculate $\rho_{n}$ based on the generated sample from BNGM model. Afterwards, we solve equation (7.2) w.r.t $\theta$.

$$
\rho_{\theta}=12 \int_{(0, \infty)^{2}}\left[\bar{F}_{\theta}(x, y)-\bar{F}_{1}(x) \bar{F}_{2}(y)\right] d \bar{F}_{1}(x) d \bar{F}_{2}(y) .
$$

\subsubsection{Tau-inversion}

The dependence measure Kendall's Tau $(\tau)$ is defined based on concordance. Let $\bar{F}_{\theta}$ be the bivariate survival function with dependence parameter $\theta$ and $\bar{F}_{1}, \bar{F}_{2}$ be its corresponding marginals, then the population version of Kendall's Tau is

$$
\tau=4 \int_{(0, \infty)^{2}} \bar{F}_{\theta}(x, y) d \bar{F}_{\theta}(x, y)-1
$$


where $\theta$ is the dependence parameter of the population. The sample version of Kendall's Tau is defined as

$$
\tau_{n}=\frac{4}{n(n-1)} Q_{n}-1
$$

such that $Q_{n}$ is the number of concordant pairs. The pairs $\left(X_{i}, Y_{i}\right)$ and $\left(X_{j}, Y_{j}\right)$ are concordant when $\left(X_{i}-X_{j}\right)\left(Y_{i}-Y_{j}\right)>0$. For the $\operatorname{BNGM}\left(\alpha_{0}, \alpha_{1}, \beta_{0}, \beta_{1}, \theta\right)$ model we estimate the marginal parameters $\left(\alpha_{0}, \alpha_{1}, \beta_{0}, \beta_{1}\right)$ based on MLE and then estimate dependence parameter $\theta$ based on Tau-inversion method. To estimate $\theta$, first, we calculate $\tau_{n}$ based on the generated sample from BNGM model. Afterwards, we solve equation (7.3) w.r.t $\theta$.

$$
\tau_{n}=4 \int_{(0, \infty)^{2}} \bar{F}_{\theta}(x, y) d \bar{F}_{\theta}(x, y)-1
$$

\subsubsection{Performance Analysis}

Now, we want to specify the finite sample performance of marginal parameters $\left(\alpha_{0}, \alpha_{1}\right.$, $\left.\beta_{0}, \beta_{1}\right)$ and dependence parameter $\theta$. The evaluation is based on bias and mean square error (MSE) of the mentioned estimators. A specific sample size $n$ has been taken from $\operatorname{BNGM}(1,1,1,1,3)$ (as an example) and estimators have been calculated based on 10000 iterations. The marginal parameters have been estimated based on MLE method and their results are given in Figure 8. We can see as the sample size increases the MSE of estimators become smaller and the values of bias become more stable near zero. The dependence parameter has been estimated with MLE, Tau-inversion and Rhoinversion methods. Their results are given in Figure 9. Clearly, the ML estimator is the best estimator for the dependence parameter $\theta$ and after that Rho-inverse estimator and Tau-inverse estimator is the worst estimator of $\theta$. Evidently, the values of bias becomes more stable around zero as the sample size increases.

We must note that For MLE, the global maximum was unique all the time and did not correspond to the boundary of the parameter space. The computational time required to identify the global maximum after trying out all combinations of the initial values did not exceed 4 hours. For Rho-inverse and Tau-inverse method, the root of the equations (7.2) and (7.3) was unique depending on the value of $\theta$ being in the interval $\left(0, \rho_{\max }(\theta)\right)$ or $\left(\rho_{\max }(\theta), \infty\right)$ for Spearman's rho and $\theta$ being in the interval $\left(0, \tau_{\max }(\theta)\right)$ or $\left(\tau_{\max }(\theta), \infty\right)$ for Kendall's tau, where $\rho_{\max }(\theta)$ and $\tau_{\max }(\theta)$ are the maximum amount of $\tau$ and $\rho$ for different values of $\theta$ (see Figure 2). 

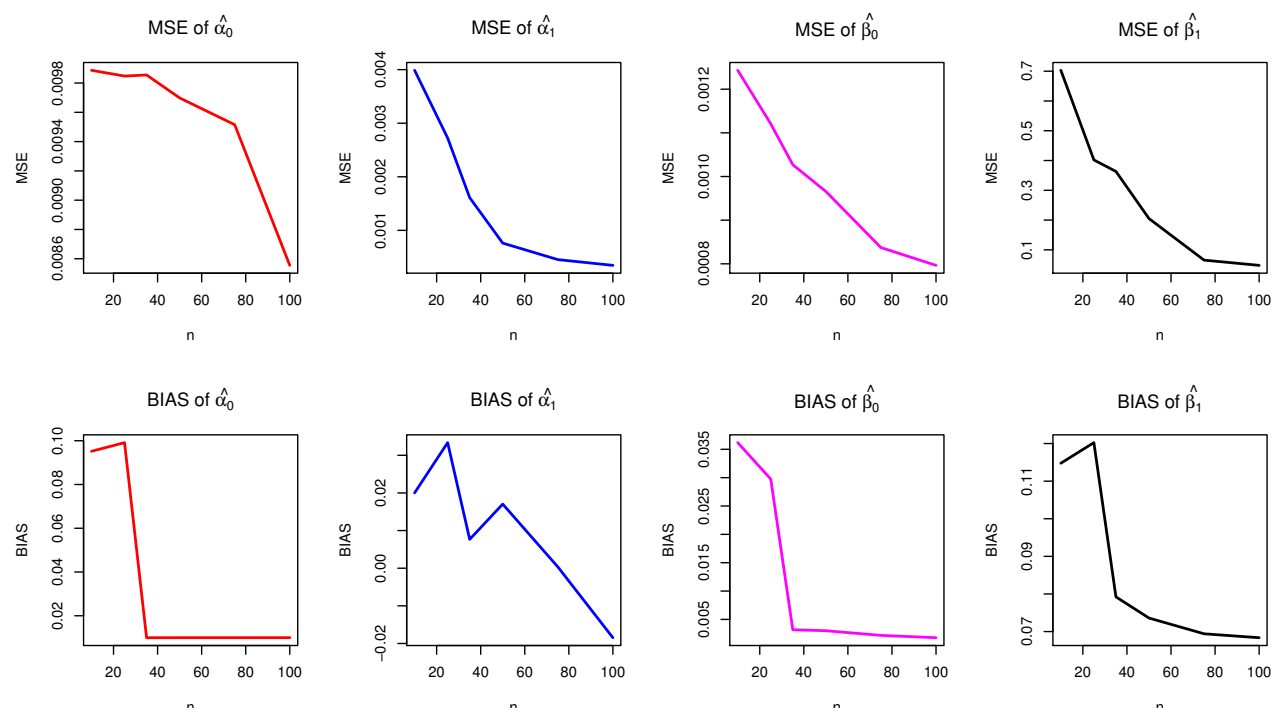

Figure 8: MSE (first row) and bias (second row) of ML estimators for marginal parameters $\left(\alpha_{0}, \alpha_{1}, \beta_{0}, \beta_{1}\right)$.
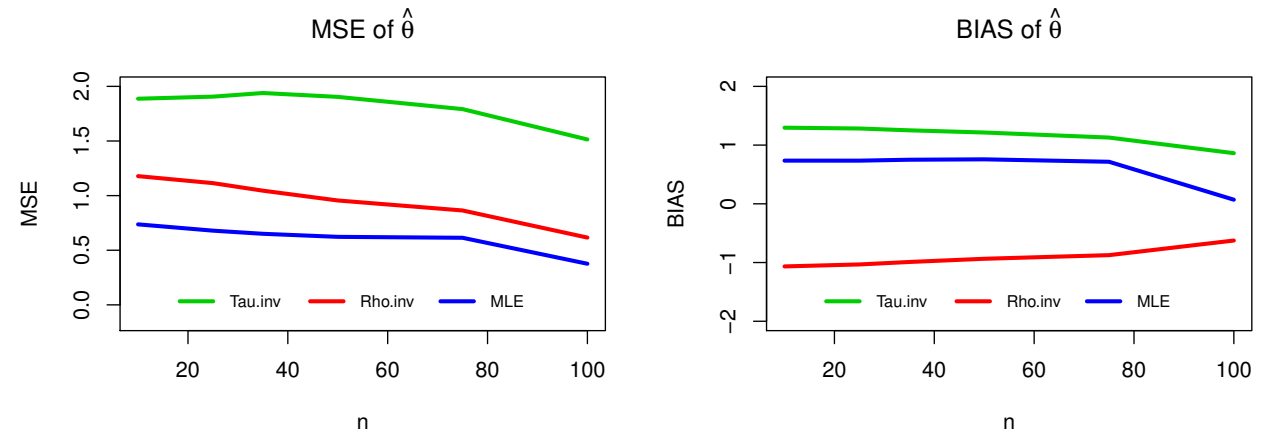

Figure 9: MSE (left) and bias (right) of three estimators (MLE, Tau-inversion and Rhoinversion) for dependence parameter $\theta$.

\section{The Multivariate Case}

Let $X_{0}^{(j)} \sim \operatorname{Exp}\left(\alpha_{0}^{(j)}\right)$ and $X_{i}^{(j)} \stackrel{i i d}{\sim} \operatorname{Exp}\left(\alpha_{1}^{(j)}\right)$ for $j=1, \ldots, n, i=1, \ldots, m_{j}$ such that $X_{i}^{(j)}$ and $X_{k}^{(p)}$ are independent for $j \neq p, i \neq k, j=p=1, \ldots, n, i=0, \ldots, m_{j}$ and $k=0, \ldots, m_{p}$. Put 
$T_{j}=\min \left\{X_{0}^{(j)}, X_{1}^{(j)}, \ldots, X_{M_{j}}^{(j)}\right\}$ where $\mathbf{M}=\left(M_{1}, \ldots, M_{n}\right)$ is distributed from a multivariate Poisson distribution introduced in Johnson et al. (1997). Let the probability generating function of multivariate Poission distribution given as

$$
A_{\mathbf{M}}\left(t_{1}, \ldots, t_{n}\right)=\exp \left\{\sum_{i=1}^{n} \theta_{i} t_{i}+\sum_{i<j} \sum_{i j} \theta_{i} t_{j}+\ldots+\theta_{12 . . n} t_{1} t_{2} \ldots t_{n}-A\right\},
$$

with $A=\sum_{i=1}^{n} \theta_{i}+\sum \sum_{i<j} \theta_{i j}+\sum \sum \sum_{i<j<k} \theta_{i j k}+\ldots+\theta_{12 . . n}$. Rewritting $A_{\mathbf{M}}$, we have

$$
\begin{aligned}
A_{\mathbf{M}}\left(t_{1}, \ldots, t_{n}\right) & =\exp \left\{-\sum_{i=1}^{n} \theta_{i}\left(1-t_{i}\right)-\sum_{i<j} \sum_{i j} \theta_{i j}\left(1-t_{i} t_{j}\right)\right. \\
& \left.-\sum \sum_{i<j<k} \sum_{i j k}\left(1-t_{i} t_{j} t_{k}\right)-\ldots-\theta_{12 . . n}\left(1-t_{1} t_{2} \ldots t_{n}\right)\right\} .
\end{aligned}
$$

Consider the case where all parameters except $\theta_{12 . . n}$ tend to zero, then we get

$$
A_{\mathbf{M}}\left(t_{1}, \ldots, t_{n}\right)=\exp \left\{-\sum_{i=1}^{n} \theta_{i}\left(1-t_{i}\right)-\theta_{12 . . n}\left(1-t_{1} t_{2} \ldots t_{n}\right)\right\} .
$$

We want to find the distribution of $\mathbf{T}=\left(T_{1}, \ldots, T_{n}\right)$ such that $\mathbf{T}$ and $\mathbf{M}$ are independent. So,

$$
\begin{aligned}
\bar{F}_{\mathbf{T}}\left(t_{1}, \ldots, t_{n}\right) & =P\left(T_{1}>t_{1}, \ldots, T_{n}>t_{n}\right), \\
& =\sum_{\mathbf{m}} P\left(X_{0}^{(1)}>t_{1}, \ldots, X_{m_{n}}^{(n)}>t_{n} \mid \mathbf{M}=\mathbf{m}\right) P(\mathbf{M}=\mathbf{m}), \\
& =\exp \left\{-\sum_{k=1}^{n} \alpha_{0}^{(k)} t_{k}\right\} \sum_{\mathbf{m}} \prod_{j=1}^{n}\left(e^{-\alpha_{1}^{(j)} t_{j}}\right)^{m_{j}} P(\mathbf{M}=\mathbf{m}), \\
& =\exp \left\{-\sum_{k=1}^{n} \alpha_{0}^{(k)} t_{k}\right\} A_{\mathbf{M}}\left(e^{-\alpha_{1}^{(1)} t_{1}}, \ldots, e^{-\alpha_{1}^{(n)} t_{n}}\right),
\end{aligned}
$$

\section{Conclusion}

In Demography literature, Golubev (2009 pp. 4) proposes the Gompertz-Makeham law for homogeneous populations as

$$
h(t)=C(t)+\Lambda \exp \{-E(t)\},
$$


where irresistible stresses are considered in $C(t)$ and resistible stresses are captured by $\Lambda \exp \{-E(t)\}$. We have extended the Gompertz-Makeham law to bivariate case where dependence parameter makes the model more flexible. Some dependence properties are derived. Also, the behaviour of conditional and bivariate hazard rate functions are investigated. Some reliability indexes such as ageing intensity and stress-strength are computed and their variation are illustrated. We plotted the variability and the flexibility of the BNGM model and presented methods to estimate the parameters. Finally, a performance analysis on the estimators of dependence parameters is given. For future studies we will focus on the multivariate version of BNGM model.

\section{Acknowledgement}

The authors would like to thank the anonymous refrees, whose comments and suggestions improved the presentation of the paper.

\section{References}

Abd El-Bar, A. M. (2018), An extended gompertz-makeham distribution with application to lifetime data. Communications in Statistics-Simulation and Computation, 47(8), 2454-2475.

Adham, S. A., and Walker, S. G. (2001), A multivariate Gompertz-type distribution. Journal of Applied Statistics. 28(8), 1051-1065.

Anderson, J. E., Louis, T. A., Holm, N. V., and Harvald, B. (1992), Time-dependent association measures for bivariate survival distributions. Journal of the American Statistical Association, 87(419), 641-650.

Bailey, R. C., and Homer, L. D. (1977), Computations for a best match strategy for kidney transplantation. Transplantation, 23(4), 329-336.

Bailey, R. C., Homer, L. D., and Summe, J. P. (1977), A proposal for the analysis of kidney graft survival. Transplantation, 24(5), 309-315.

Bebbington, M., Green, R., Lai, C. D., and Zitikis, R. (2014), Beyond the Gompertz law: exploring the late-life mortality deceleration phenomenon. Scandinavian Actuarial Journal, 2014(3), 189-207. 
Clayton, D. G. (1978), A model for association in bivariate life tables and its application in epidemiological studies of familial tendency in chronic disease incidence. Biometrika, 65(1), 141-151.

Denuit, M., Dhaene, J., Goovaerts, M., and Kaas, R. (2006), Actuarial theory for dependent risks: measures, orders and models. John Wiley \& Sons.

El-Sherpieny, E. A., Ibrahim, S. A., and Bedar, R. E. (2013), A new bivariate distribution with generalized Gompertz marginals. Asian Journal of Applied Sciences, 1(04), 141-150.

Feng, X., and He, G. (2008), Estimation of parameters of the Makeham distribution using the least squares method. Mathematics and Computers in Simulation, 77(1), 34-44.

Golubev, A. (2009), How could the Gompertz-Makeham law evolve. Journal of theoretical Biology, 258(1), 1-17.

Gompertz, B. (1825), XXIV. On the nature of the function expressive of the law of human mortality, and on a new mode of determining the value of life contingencies. In a letter to Francis Baily, Esq. FRS \& c. Philosophical transactions of the Royal Society of London, 115, 513-583.

Jiang, R., Ji, P., and Xiao, X. (2003), Aging property of unimodal failure rate models. Reliability Engineering E System Safety, 79(1), 113-116.

Johnson, N. L., and Kotz, S. (1975), A vector multivariate hazard rate. Journal of Multivariate Analysis, 5(1), 53-66.

Johnson, N., Kotz, S., and Balakrishnan, N. (1994), Continuous Univariate Distributions. Volume 1, 2nd Edition. John Wiley and Sons, New York.

Johnson, N. L., Kotz, S., and Balakrishnan, N. (1997), Discrete multivariate distributions. Volume 165. Wiley. John Wiley and Sons (New York).

Juckett, D. A., and Rosenberg, B. (1993), Comparison of the Gompertz and Weibull functions as descriptors for human mortality distributions and their intersections. Mechanisms of Ageing and Development, 69(1-2), 1-31.

Kolev, N. (2016), Characterizations of the class of bivariate Gompertz distributions. Journal of Multivariate Analysis, 148, 173-179.

Lai, C. D., and Xie, M. (2006), Stochastic ageing and dependence for reliability. Springer Science \& Business Media. 
Marshall, A. W., and Olkin, I. (1997), A new method for adding a parameter to a family of distributions with application to the exponential and Weibull families. Biometrika, 84(3), 641-652.

Marshall, A. W., and Olkin, I. (2007), Life distributions. Springer, New York.

Marshall, A. W., and Olkin, I. (2015), A bivariate Gompertz-Makeham life distribution. Journal of Multivariate Analysis, 139, 219-226.

Melnikov, A., and Romaniuk, Y. (2006), Evaluating the performance of Gompertz, Makeham and Lee-Carter mortality models for risk management with unit-linked contracts. Insurance: Mathematics and Economics, 39(3), 310-329.

Missov, T. I., and Lenart, A. (2013), Gompertz-Makeham life expectancies: expressions and applications. Theoretical Population Biology, 90, 29-35.

Nelsen, R. B. (2007), An introduction to copulas. Springer Science \& Business Media.

Oakes, D. (1989), Bivariate survival models induced by frailties. Journal of the American Statistical Association, 84(406), 487-493.

Scollnik, D. P. M. (1995), Simulating random variates from Makeham's distribution and from others with exact or nearly log-concave densities. Transactions of the Society of Actuaries, 47, 409-437.

Shih, J. H., and Emura, T. (2019), Bivariate dependence measures and bivariate competing risks models under the generalized FGM copula. Statistical Papers, 60(4), 1101-1118.

Szymkowiak, M. (2020), Lifetime Analysis by Aging Intensity Functions. Springer International Publishing.

Wang, J. L., Muller, H. G., and Capra, W. B. (1998), Analysis of oldest-old mortality: Life-tables revisited. The Annals of Statistics, 26(1), 126-163. 